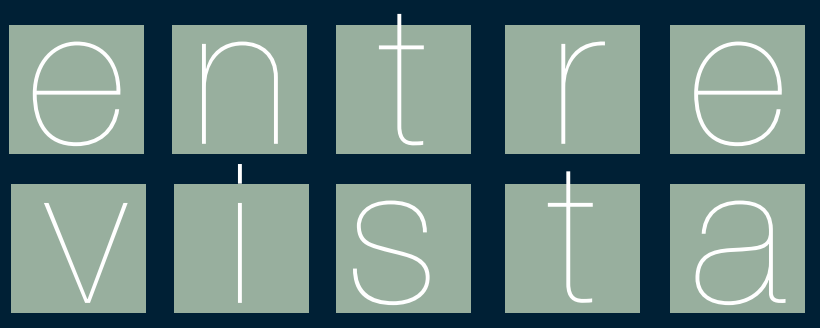




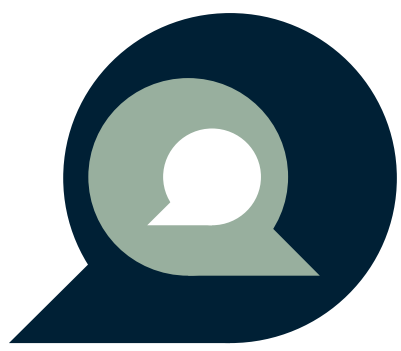




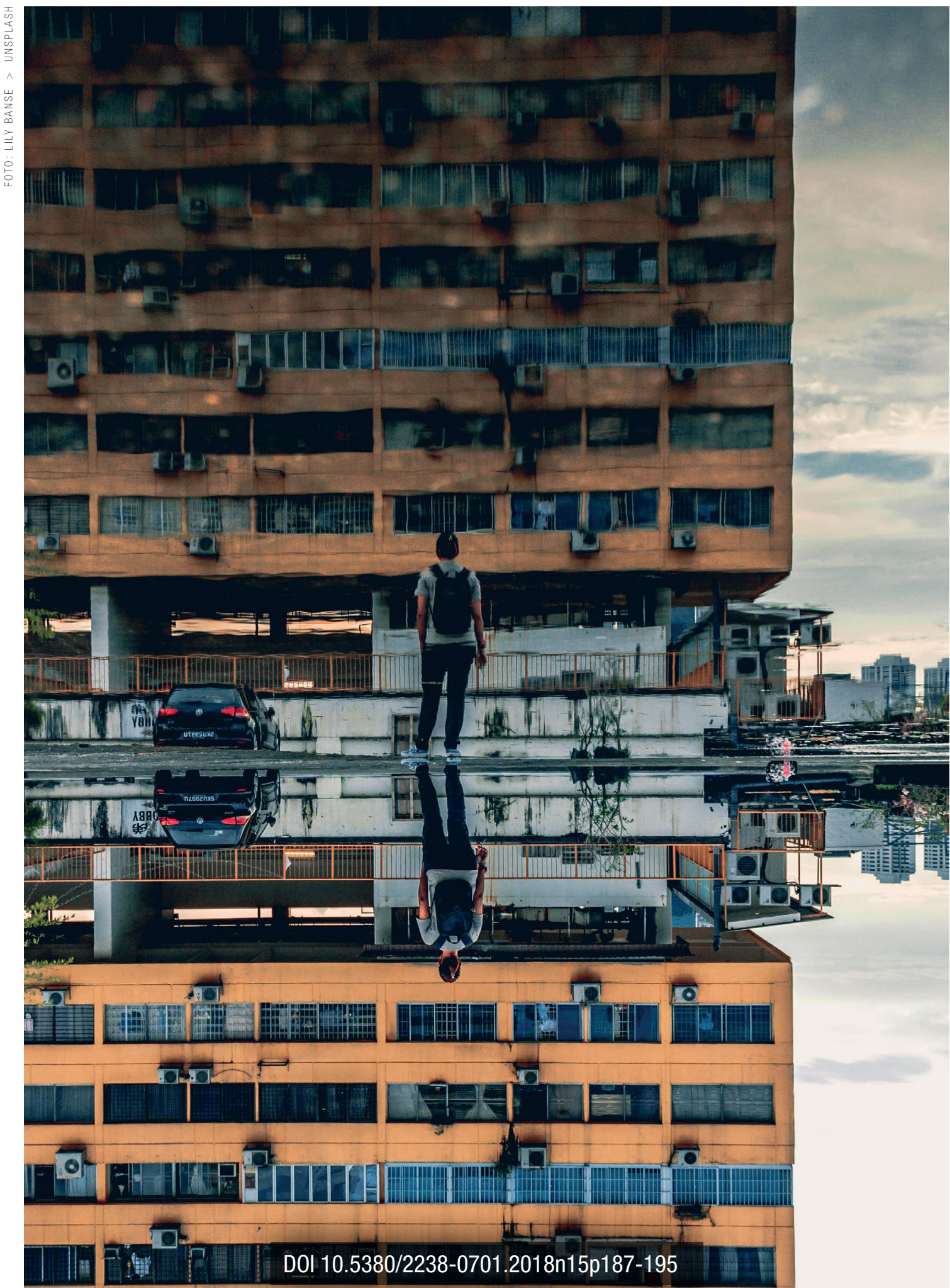


Educação Superior. Relações Brasil-Argentina. Ciências da Comunicação.

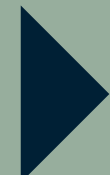




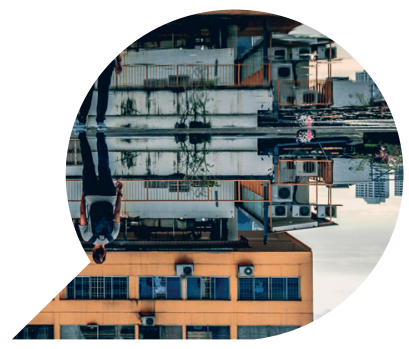

\section{Cooperação Sul-Sul: um desafio que deve unir Brasil e Argentina}

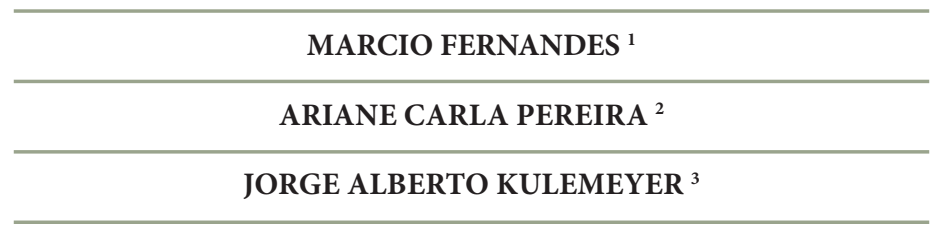

Resumo: Esta entrevista apresenta as visões de Rodolfo Alejandro Tecchi (reitor da Universidad Nacional de Jujuy/UNJu/Argentina), sobre as relações acadêmicas entre Brasil e Argentina, incluindo aspectos das Ciências da Comunicação. Entre o primeiro trimestre de 2017 e o primeiro trimestre de 2018, Tecchi presidiu o Consejo Interuniversitário Nacional (CIN), entidade que reúne as mais de 60 universidades públicas do país vizinho e um dos mais importantes órgãos colegiados da Educação Superior na América Latina. Entre outros pontos debatidos com o reitor, está a predileção de muitos pesquisadores brasileiros e argentinos em buscar cooperação somente no sentido Sul-Norte, deixando de lado as potencialidades com os vizinhos sobretudo dos países do Mercosul. E também o papel das Ciências Sociais e Humanas na sociedade contemporânea.

Palavras-Chave: Relações binacionais; Realidades sociais; Ciências da Comunicação.

1 Doutor em Comunicação e Cultura pela Universidade Federal do Rio de Janeiro (UFRJ), Chefe de Gabinete da Reitoria da Universidade Estadual do Centro-Oeste (Unicentro).

2 Doutora em Comunicação e Cultura, Universidade Federal do Rio de Janeiro (UFRJ). Diretora de Imprensa da Unicentro.

3 Doutor em Ciências Filosóficas, Universidade de Colônia (Alemanha). Diretor do Centro de Investigaciones sobre Cultura y Naturaleza Andinas (CICNA)/Universidad Nacional de Jujuy (UNJu/ Argentina). 
Ação Midiática (AM): No Brasil, em geral, as investigações científicas com Europa e com Estados Unidos costumam ser mais valorizadas do que aquelas que podem ser concretizadas com nossos vizinhos da América Latina. Isso também ocorre na Argentina?

Rodolfo Alejandro Tecchi (RAT): Em geral, podemos dizer que a valorização Sul-Norte aqui na Argentina é bastante similar com a que ocorre no Brasil.

Ação Midiática (AM): Quais são as razões para que esse seja o cenário?

RAT: No âmbito dos processos históricos de dependência, em nossos países se instalou a ideia de que a atividade científica que se realiza nos países chamados de "centrais" é mais importante ou mais avançada do que aquela que se realiza em nossas instituições. Obviamente, aqueles países (da Europa e dos Estados Unidos) querem ter o controle de quais são as linhas de investigação nas quais devem se desenvolver a Investigação, o Desenvolvimento e a Inovação.

Ação Midiática (AM): Brasil e Argentina compartilham um importante trecho de fronteira. Por quais razões o senhor que crê que há existam tão poucos estudos conjuntos sobre temas fronteiriços, como identidades regionais comuns (na Música, na Língua, nas Comunicações, etc)?

RAT: Temos de assumir publicamente que nossas universidades, principalmente aquelas instaladas nas proximidades de ambos os lados da fronteira Brasil-Argentina, não têm realizado um esforço concreto nesse sentido. De fato, é uma obra pendente de realização.

Ação Midiática (AM): Embora dados econômicos e indicadores sociais apontem tanto a importância dos setores industriais como das atividades culturais em nossos países enquanto agentes de melhoria da vida das populações, para grande parte da sociedade contemporânea há a ideia de que a Ciência ocorre somente em algumas áreas, como Saúde, Engenharias e Biologia. Ou seja, as investigações em Ciências Sociais e 
Humanas não seriam importantes. Como é a sua visão a respeito disso, em nível pessoal, e como o CIN vê esse tema?

RAT: Voltemos à primeira pergunta. Há interesses concretos para que nosso esforço em Investigação, Desenvolvimento e Inovação sejam voltados para as áreas biomédicas e exatas, por exemplo. Nesse cenário, há quem defenda que os temas sociais são menos importantes, justamente pelo fato de que esses tópicos focados nos problemas de nossas comunidades do Sul não são considerados relevantes pelos países chamados "centrais".

Ação Midiática (AM): O Estado brasileiro do Paraná está avançando bastante na cooperação científica com várias zonas da Argentina, em áreas como Comunicação Social, Ciências Sociais e História, em especial com as Universidades Nacionais (Federais) de Misiones, Salta e de Jujuy. É possível pensar no lançamento de editais públicos apoiados pelo CIN para fomentar projetos conjuntos entre Paraná e os Estados argentinos do Norte e do Oeste (chamados de Litorâneos, por conta do rio Paraná)? Quais seriam os outros possíveis agentes financiadores de iniciativas como essa?

RAT: Seria muito relevante poder crescer nesse sentido. E os Governos dos nossos Estados nacionais e regionais deveriam contribuir decisivamente para o desenvolvimento de ações cooperadas.

Ação Midiática (AM): A política do Conicet (equivalente ao CNPq) de implantar Unidades Executoras Regionais é considerada bastante válida por muitos investigadores brasileiros que atuam em associação com instituições argentinas. Qual seria a visão do CIN acerca da possibilidade de se instalar uma Unidade Executora nessas zonas de frontera com o Brasil que se dedicasse a temas de interesse comum das duas nações?

RAT: Nós temos a experiencia da Universidade Nacional de Jujuy (UNJu). Em apenas três anos, temos constituído seis grandes projetos com o Conicet, em temas como Lítio, Ecossistemas de Montanha, Desenvolvimento Social, Setor de alimentos, Arqueometría e Energias 
Sustentáveis. Em todos esses eixos, estamos abertos à cooperação internacional, particularmente com os países vizinhos.

Ação Midiática (AM): Para que o leitor brasileiro possa saber mais sobre o CIN, como funciona o dia a dia da entidade?

RAT: O CIN está previsto na Lei de Educação Superior argentina, que establece un conselho de universidade públicas e outro para as de gestão privada. O CIN reúne os reitores de aproximadamente sessenta universidades federais e estaduais. Suas autoridades são eleitas anualmente. Temos um presidente, um vice-presidente e diversos responsáveis por comissões, dentre elas Assuntos académicos, Credenciamento de cursos, Ciência e Tecnologia, Extensão, Relações Internacionais, Orçamento, Relações Institucionais, Conectividade e Vinculação Tecnológica, Pós-graduação e Meios de Comunicação.

Ação Midiática (AM): O CIN mantém algumas chamadas públicas, como as bolsas do Programa Estratégico de Formação de Recursos Humanos em Investigação e Desenvolvimento. Como tem sido a adesão de investigadores de Ciências Sociais e Humanidades?

RAT: Ao menos 1/3 dessas bolsas se dedicam a esses dois campos.

Ação Midiática (AM): Não faz muito e o CIN firmou convênios gerais com entidades latinas do porte de ASCUN (Colômbia), ANUIES (México) e ABRUEM (Brasil), além de ter cooperação com a ANDIFES (Brasil). De que maneiras esses acordos podem ajudar a modificar a realidade social dos países da América Latina?

RAT: O objetivo é avançar até um programa único de mobilidade de estudantes e de professores para América Latina e Caribe. Isso será uma contribuição muito importante para formar dirigentes, investigadores e estudantes comprometidos com nossas realidades regionais.

Ação Midiática (AM): Ação Midiática é uma revista da área de Comunicação Social. Nesse sentido, que ideias e conselhos poderiam 
ser passados a investigadores de Comunicação do Brasil no sentido de desenvolver produtos, processos e patentes realmente inovadoras, que beneficiem diretamente a sociedade?

RAT: A Comunicação em nossos países é um espaço crítico no qual as universidades devem colaborar para que todas as vozes de nossas comunidades possam ser escutadas. Se não garantirmos isso com segurança, a Comunicação ficará em poucas mãos e isso não é democrático.

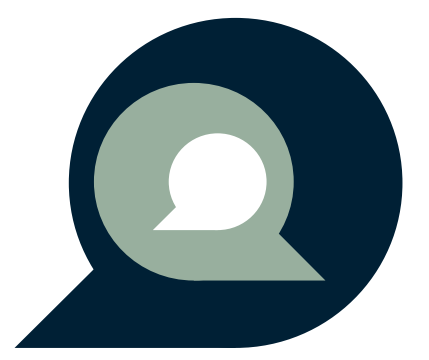




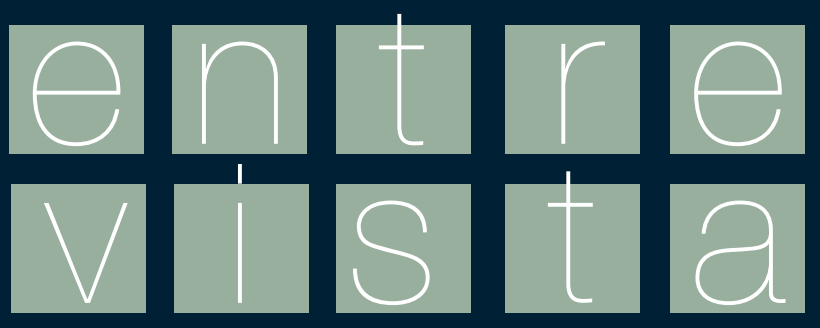




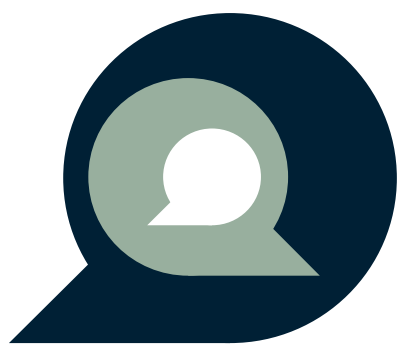




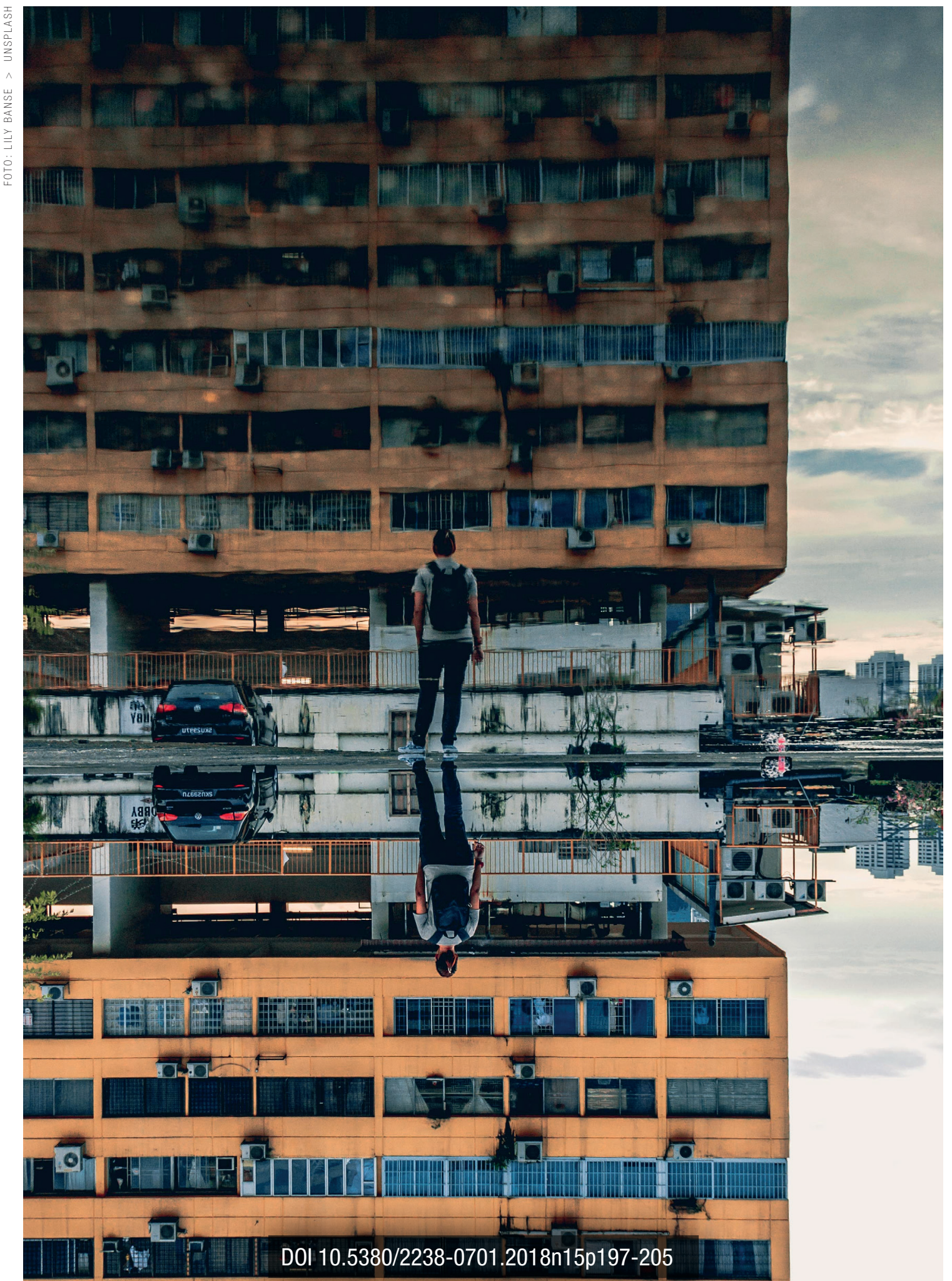


Educación Superior. Relaciones Brasil-Argentina. Ciencias de la Comunicación. 


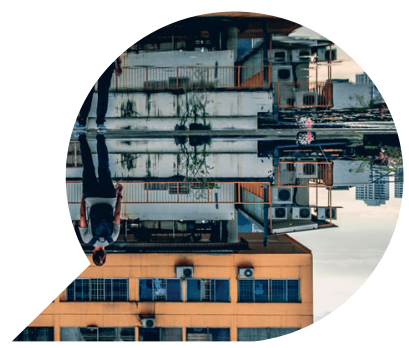

\section{Cooperación Sur-Sur: hacia un desafío que debe unir Brasil y Argentina}

\section{MARCIO FERNANDES ${ }^{1}$}

\begin{tabular}{c}
\hline ARIANE CARLA PEREIRA $^{2}$ \\
\hline JORGE ALBERTO KULEMEYER $^{3}$ \\
\hline
\end{tabular}

Resumen: Esa entrevista presenta las visiones de Rodolfo Alejandro Tecchi (rector de la Universidad Nacional de Jujuy/UNJu/Argentina), acerca de las relaciones académicas entre Brasil y Argentina, incluyendo aspectos de las Ciencias de la Comunicación. Entre el primer trimestre de 2017 y el primer trimestre de 2018, Tecchi ha presidido el Consejo Interuniversitario Nacional (CIN), entidad que agrega las más de 60 universidades públicas de la nación vecina y uno de los más prestigiosos institutos colegiados de Educación Superior en Latinoamérica. Entre otros puntos debatidos con el rector, está el deseo primero de muchos investigadores brasileños y argentinos en tener cooperación básicamente en el sentido Sur-Norte, dejando en según plano las potencialidades con los países sobre todo del Mercosur. Y también el rol de las Ciencias Sociales e de Humanidades en la sociedad contemporánea.

Palabras clave: Relaciones Brasil-Argentina; Realidades sociales; Ciencias de la Comunicación.

1 Doutor em Comunicação e Cultura pela Universidade Federal do Rio de Janeiro (UFRJ), Chefe de Gabinete da Reitoria da Universidade Estadual do Centro-Oeste (Unicentro).

2 Doutora em Comunicação e Cultura, Universidade Federal do Rio de Janeiro (UFRJ). Diretora de Imprensa da Unicentro.

3 Doutor em Ciências Filosóficas, Universidade de Colônia (Alemanha). Diretor do Centro de Investigaciones sobre Cultura y Naturaleza Andinas (CICNA)/Universidad Nacional de Jujuy (UNJu/ Argentina). 
Ação Midiática (AM): En Brasil, en general, las investigaciones científicas con Europa y Estados Unidos son más valorizadas que las que se llevan a cabo con nuestros vecinos de Latino América. ¿Es así también en Argentina?;

RAT: En general podemos decir que la valorización es similar a la que se le otorga en Brasil

Ação Midiática (AM): ¿Cuáles las razones para que así sea?

RAT: En el marco de los procesos de dependencia, en nuestros países se instaló la idea de que la actividad científica que se realiza en los países llamados precisamente "centrales" es más importante o avanzada que la que se realiza en nuestras instituciones. Obviamente aquellos países quieren tener el control de cuales son las líneas de investigación en las cuales se desenvuelven la Investigación, en el Desarrollo Científico y en la Innovación.

Ação Midiática (AM): Argentina y Brasil comparten un tramo importante de frontera. ¿Por qué cree usted que hay muy pocos estudios conjuntos sobre los temas fronterizos, como identidades regionales comunes (en la música, en la lengua, comunicaciones, etc)?

RAT: Tenemos que asumir que probablemente nuestras universidades, principalmente aquellas ubicadas cerca de ambos lados de la frontera no han realizado un esfuerzo concreto en este sentido. $Y$ es una asignatura pendiente.

Ação Midiática (AM): A pesar del peso que, medido en términos de PBI, muestran en la Economía de nuestros países las industrias y actividades culturales y de la necesidad de comprensión para su mejor atención de las problemáticas sociales, para gran parte de la sociedad hoy la idea de Ciencia esta muy enlazada con solamente algunas áreas, como Salud, Ingenierías y Biología. O sea, las investigaciones en Ciencias Sociales y Humanidades no serían importantes. ¿Cómo es la visión al respecto en su mirada y también en el ámbito del CIN? 
RAT: Volvemos a la pregunta inicial. Hay intereses concretos para que nuestro esfuerzo en $I+D-i$ se oriente a las biomédicas, y a las exactas, por ejemplo. En ese marco importan menos los temas sociales porque precisamente estos tópicos que se enfocan en problemáticas de nuestras comunidades no son trascendentes para los países "centrales".

Ação Midiática (AM): La Provincia brasileña de Paraná está avanzando mucho en la cooperación científica con varias zonas de Argentina, en áreas como Comunicación Social, Ciencias Sociales e Historia, en especial con las Universidades Nacionales de Misiones, Salta y Jujuy. ¿Es posible pensar el lanzamiento de convocatorias públicas apoyadas por CIN para fomentar proyectos conjuntos entre Paraná y Provincias del Norte y del Litoral de Argentina? Cual serian los posibles agentes auspiciadores de iniciativas así?

RAT: Sería muy auspicioso poder crecer en este sentido. Y nuestros Estados nacionales y regionales deberían contribuir especialmente para su desarrollo.

Ação Midiática (AM): La política de Conicet de implantar Unidades Ejecutoras Regionales es considerada muy válida por muchos investigadores brasileños que actúan en asociación con instituciones argentinas. ¿Cuál la visión del CIN acerca de la posibilidad de se implantar una Unidad Ejecutora en las zonas de frontera con Brasil, para se dedicar a temas de interés común a las dos naciones?

RAT: Nosotros tenemos la experiencia de la Universidad Nacional de Jujuy. En solo tres años hemos constituido seis instituciones con el Conicet, en temas tales como litio, ecosistemas de altura, desarrollo social, alimentos, arqueometría, y energias sustentables. En todos estos temas estamos abiertos a la cooperación internacional, particularmente con los países vecinos.

Ação Midiática (AM): Para que el público brasileño pueda saber más, ¿cómo funcionan en el día a día del CIN?

RAT: El CIN está consagrado por la Ley de Educación Superior que establece 
un consejo para universidades públicas estatales y otro para las de gestión privada. Reúne a los rectores de unas sesenta universidades federales $y$ estaduales. Elige sus autoridades anualmente. Se trata de un Presidente, un Vicepresidente y distintos responsables de comisiones: asuntos académicos, acreditación, ciencia y tecnología, extensión, relaciones internacionales, presupuesto, relaciones institucionales, conectividad y vinculación tecnológica, posgrado y medios de comunicación.

Ação Midiática (AM): El CIN mantiene algunas convocatorias, como las Becas del Programa Estratégico de Formación de Recursos Humanos en Investigación y Desarrollo. ¿Cuál esta siendo la adhesión de los investigadores de Ciencias Sociales y Humanidades?

RAT: Al menos un tercio de estas becas se dedican a estos temas.

Ação Midiática (AM): Hace poco, CIN subscribió convenios marcos con entidades latinas como ASCUN (Colombia), ANUIES (México) y ABRUEM (Brasil), además de tener cooperación también con ANDIFES (Brasil). ¿De que maneras eses acuerdos podrán ayudar a cambiar la realidad social de nuestros países de Latino América?

RAT: El objetivo es avanzar hacia un programa de movilidad de estudiantes y profesores para América Latina y el Caribe. Esto será una contribución fundamental para formar dirigentes, investigadores, $y$ académicos comprometidos con nuestras realidades.

Ação Midiática (AM): Ação Midiática es una revista de área de Comunicación Social. En ese marco, que ideas y consejos se podría subministrar para investigadores de Comunicación de Brasil en el sentido de desarrollar productos, técnicas y patentes realmente innovadoras, que beneficien la sociedad directamente?

RAT: La comunicación en nuestros países en un espacio críticos en el cual las Universidades debemos colaborar para que todas las voces de nuestras comunidades puedan ser escuchadas. Si no estuviéramos en esta tarea seguramente la comunicación quedaría en pocas manos, y eso no es democrático. 


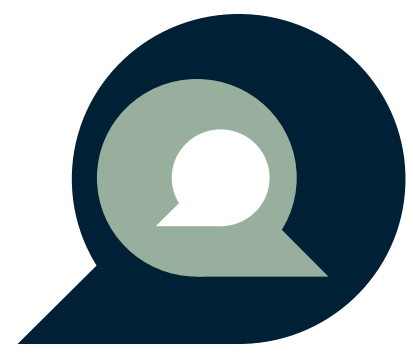

